\title{
Representações sociais sobre pé diabético: contribuições para Atenção Primária à saúde no Nordeste brasileiro
}

\author{
Social representations on diabetic foot: contributions to PHC \\ in the Brazilian Northeast
}

Geysa Santos Góis Lopes (https://orcid.org/0000-0002-6801-1940) ${ }^{1}$

Isaura Leticia Tavares Palmeira Rolim (https://orcid.org/0000-0002-8453-2543) ${ }^{2}$

Renata de Sousa Alves (https://orcid.org/0000-0003-0630-1499) ${ }^{3}$

Talitha Rodrigues Ribeiro Fernandes Pessoa (https://orcid.org/0000-0001-8254-0876) ${ }^{4}$

Evanira Rodrigues Maia (https://orcid.org/0000-0001-9377-7430) ${ }^{5}$

Maria do Socorro Vieira Lopes (https://orcid.org/0000-0003-1335-5487) ${ }^{5}$

Ana Patrícia Pereira Morais (https://orcid.org/0000-0001-6188-7897) ${ }^{6}$

Rejane Christine de Sousa Queiroz (https://orcid.org/0000-0003-4019-2011) ${ }^{7}$

\footnotetext{
${ }^{1}$ Rede Sarah de Hospitais do Aparelho Locomotor. Avenida Governador Luiz Rocha s/n, Liberdade 65035-270 São Luís MA Brasil.

geysagois@hotmail.com ${ }^{2}$ Departamento de

Enfermagem, Universidade Federal do Maranhão. São

Luís MA Brasil.

${ }^{3}$ Departamento de Análises

Clínicas e Toxicológicas,

Universidade Federal do

Ceará. Fortaleza CE Brasil.

${ }^{4}$ Departamento de

Odontologia Clínica e

Social, Universidade Federal

da Paraíba. João Pessoa PB

Brasil.

${ }^{5}$ Universidade Regional do

Cariri. Crato CE Brasil.

${ }^{6}$ Universidade Estadual do

Ceará, Departamento de

Enfermagem. Fortaleza CE

Brasil.

${ }^{7}$ Departamento de Saúde

Pública, Universidade

Federal do Maranhão. São

Luís MA Brasil.
}

\begin{abstract}
This study aimed to identify the structuring elements guiding the establishment of the social representations of diabetic foot among people with diabetes mellitus. This qualitative study is based on the Social Representations Theory and was conducted in a capital of the Brazilian Northeast. The free word association test and a roadmap were used to characterize the sociodemographic and clinical profile to collect data. The analysis was performed using openEVOC software. The constituent elements of the diabetic foot's social representation "cure" and "really bad", revealing that living with a diabetic foot is challenging, but there is hope for a cure, which is a driving force in the daily search for care. The "prevention" element emerged in the representational field, denoting a more critical view and a capacity to transform the identified core elements. We found that the representational structure is based on subjective, valuating, and attitudinal contents. This knowledge can contribute to the design of interventions in the provision of care and diabetic foot screening in PHC services.
\end{abstract}

Key words Diabetic foot, Social psychology, Diabetes mellitus
Resumo A investigação tem por objetivo identificar os elementos estruturantes que orientam a formação das representações sociais do pé diabético entre pessoas com diabetes mellitus. Estudo qualitativo, fundamentado na Teoria das Representações Sociais, seguindo a vertente estrutural complementar, realizado em uma capital do Nordeste brasileiro, de fevereiro a maio de 2019, com a participação de 100 pessoas com pé diabético. Para a coleta dos dados, utilizou-se o teste de associação livre de palavras e o roteiro para caracterização do perfil sociodemográfico e clínico. A análise foi realizada por meio do software openEVOC. Os elementos constituintes da representação social do pé diabético referem-se às evocações são "cura" $e$ "muito ruim", e revelam que viver com pé diabético é desafiador, mas que há uma esperança de cura que se constitui em mola propulsora na busca cotidiana do cuidado. O elemento prevenção apareceu no campo representacional, o que denota uma visão mais crítica, podendo transformar os elementos nucleares identificados. Constata-se que a estrutura representacional encontra-se ancorada em conteúdos subjetivos, valorativos e atitudinais. Conclui-se que este conhecimento pode contribuir com o desenho de intervenções que auxiliem nas práticas de cuidados e rastreamento do pé diabético nos serviços ofertados na Atenção Primária à Saúde.

Palavras-chave Pé diabético, Psicologia social, Diabetes Mellitus 


\section{Introdução}

Ações e serviços ofertados na Atenção Primária à Saúde (APS), principalmente pelas equipes da Estratégia Saúde da Família (ESF) aos usuários com diabetes, podem contribuir para a diminuição do número de hospitalizações por complicações desta doença, incluindo o pé diabético. Tais ações visam prevenir ou conter lesões ao promover o acesso e a integralidade do cuidado em tempo oportuno para a intervenção.

Pessoas com diabetes mellitus (DM) devem ser avaliadas de maneira integral e de forma periódica, com o objetivo de detectar precocemente alterações que confiram um risco aumentado para o desenvolvimento de úlceras e outras complicações do pé diabético. Existem fluxogramas de atendimento na atenção primária e especializada em saúde que levam em conta a estratificação do risco e permitem, assim, o uso mais eficiente e efetivo de tempo e recursos das equipes ${ }^{1}$.

Pé diabético é um termo genérico que se refere à variedade de condições patológicas associadas a anormalidades neurológicas e vários graus de doença vascular periférica no membro inferior que podem afetar os pés de pacientes com DM. Constitui-se relevante problema de saúde pública, tanto nos países desenvolvidos quanto nos em desenvolvimento devido a sua frequência e a sua associação à alta mortalidade, além dos custos elevados para os serviços de saúde. Apesar da existência de diretrizes bem consolidadas mundialmente, o gerenciamento do pé diabético é um enorme desafio ${ }^{2-4}$.

Entretanto, há uma lacuna a ser preenchida por evidências científicas que apoiem boa parte da prática clínica de rotina, o que produz alguns modelos de cuidados suscetíveis às opiniões e crenças das pessoas. Tal fato pode cooperar e justificar a distribuição variável dessa condição geograficamente. Sobre o impacto populacional, identificou-se, a partir de uma revisão sistemática com metanálise em grande escala, que a prevalência global de pé diabético foi de $6,3 \%$. Tão problemático quanto esse dado é a estimativa de que $25 \%$ dos diabéticos que vivem em países em desenvolvimento apresentem, ao longo da vida, uma lesão no pé, cujo prognóstico é influenciado pela doença vascular e pela infecção, ambas associadas em mais de 50\% das amputações de membros inferiores ${ }^{4-7}$.

Estudo prévio mostrou que o indicador de qualidade de vida relacionada à saúde é marcadamente ruim em pessoas com diabetes, assinalando que o pé diabético contribui para que as pessoas acometidas experimentem severas restrições na vida diária. Destaca-se o isolamento social como consequência da mobilidade reduzida, exigência do tratamento clínico frequente e cuidado constante, com impacto na percepção de sentimentos negativos e estreita relação com níveis mais altos de depressão e pior adaptação psicossocial à doença. Estes desfechos colaboram para a existência de um cenário singular para a concepção de representações. Assim, conhecer as representações sociais (RS) sobre o pé diabético poderá auxiliar no entendimento de como as experiências cotidianas das pessoas com DM são influenciadas por essas RS e como elaboram crenças, significados, opiniões e atitudes voltadas para os cuidados dos seus pés, o que amplia a compreensão do fenômeno pé diabético ${ }^{8}$.

Outros estudos revelam que a ausência de realização do exame dos pés de portadores de diabetes, juntamente com a carência de ações educativas orientadoras e de prevenção, pressupõe aumento no risco de lesões, especialmente em pacientes com maior vulnerabilidade social ${ }^{9-11}$. Desta forma, a necessidade de acesso a práticas educativas e preventivas de cuidado, a atenção integral aos portadores de diabetes, a busca ativa e acompanhamento longitudinal desses usuários, assim como coordenação do cuidado dos pacientes com DM na rede de atenção, apontam para a fundamental importância da APS na atenção e prevenção do pé diabético de maneira ainda mais acentuada em regiões como o Nordeste brasileiro ${ }^{9-11}$.

De modo geral, mediante dois processos sociocognitivos - a ancoragem e a objetificação - as RS têm a finalidade de tornar familiar algo que não é. Ao conectar conhecimentos e conceitos novos a valores e ideias prévias e internalizadas na cultura, almejam a construção de um contexto comum a um grupo social e se revelam como elementos cognitivos, imagens, conceitos, categorias e teorias ${ }^{12}$.

Neste estudo, aplicou-se a vertente estrutural complementar, a fim de conhecer o núcleo central da RS, com fulcro na Teoria do Núcleo Central das RS proposta por $\mathrm{Abric}^{13}$, um construto teórico-metodológico que agrega a Teoria das Representações Sociais (TRS), originada por Moscovici ${ }^{12}$. O autor fundamentou-se na hipótese de que toda RS se organiza tanto em torno de um núcleo central que confere significado à representação quanto em torno de um sistema periférico que constitui a interface entre o núcleo central e a realidade concreta ${ }^{12,13}$.

O conhecimento sobre as RS tem sido útil para ampliar a compreensão do processo saúde- 
doença, ao contribuir para o desenvolvimento de abordagens arrojadas em relação às práticas terapêuticas e educativas, tão fundamentais para o gerenciamento de comorbidades como o pé diabético, por meio de condutas mais humanizadas e personalizadas, o que contribui para uma aproximação ao contexto da pessoa com diabetes ${ }^{14,15}$.

Objetiva-se identificar os elementos estruturantes que orientam a formação das representações sociais do pé diabético entre pessoas com DM.

\section{Método}

\section{Tipo de estudo}

Trata-se de uma pesquisa descritiva com abordagem qualitativa, fundamentada na Teoria das Representações Sociais (TRS), com abordagem da Teoria do Núcleo Central. Embora não seja objetivo do estudo, alguns dados quantitativos sobre os participantes foram utilizados como suporte para subsidiar as discussões, o que reitera a visão da TRS de que as ideias, crenças e valores apoiam-se na identidade dos grupos de pertença.

\section{População e cenário}

A investigação foi realizada no período de fevereiro a maio de 2019 e teve como cenário o ambulatório de endocrinologia e diabetes e as enfermarias de um hospital universitário, assim como um centro de especialidades médicas e diagnóstico, ambos localizados em uma capital do Nordeste do Brasil. A escolha dos locais para execução do estudo deve-se ao fato de serem serviços de referência no município para acompanhamento de pessoas com DM, inclusive com encaminhamentos de usuários pela APS/ESF.

Os participantes foram selecionados por meio de amostragem não probabilística por conveniência, e levou-se em consideração a disponibilidade de estar presente no local no momento da coleta de dados. Os participantes do estudo atenderam aos seguintes critérios de inclusão: ter diagnóstico de DM, há mais de 5 anos, com pé diabético; ter idade igual ou superior a 18 anos; e ter condições psicológicas e de comunicação para responder à pergunta norteadora deste estudo. Vale ressaltar que a determinação do tempo de diagnóstico de 5 anos deve-se ao conhecimento de que os significados são engendrados na experimentação da convivência. Assim, participantes com maior tempo de diagnóstico puderam con- tribuir de modo mais amplo com a proposta do estudo.

Foram excluídos do estudo pessoas com diagnóstico atual de pé diabético que tivessem parte ou a totalidade de um dos membros inferiores amputados ou que apresentassem instabilidade clínica no momento da abordagem para a coleta de dados. Optou-se por incluir na amostra apenas pessoas sem amputação para se conhecer as RS a partir da perspectiva de quem tem uma comorbidade com alto potencial de perda do membro inferior, bem como o significado disso para essas pessoas. Ademais, decidiu-se explorar a temática com as pessoas que ainda não experimentaram o processo de amputação, uma vez que os estudos qualitativos identificados na literatura não fizeram essa diferenciação.

Durante o recrutamento dos participantes, foram abordadas 110 pessoas, entretanto, dez foram excluídas por apresentarem parte de um dos membros inferiores amputados, especialmente amputação de um ou mais pododáctilos ou amputações de meio pé.

\section{Coleta de dados}

Nos atendimentos ambulatoriais, inicialmente, a pesquisadora se integrava aos pacientes na sala de espera, interagindo e buscando compreender a dinâmica dos atendimentos; com isso, buscou-se socializar com eles e participar de conversas triviais, a fim de se obter uma maior aproximação com os pacientes. Esta maneira de agir é aconselhada quando se utiliza como referencial teórico a TRS, pois é uma tática válida e imprescindível estabelecer um envolvimento pregresso com o grupo com que se intenciona desenvolver o estudo, com a finalidade de se familiarizar com os participantes.

Realizou-se teste piloto com o intuito de verificar a confiabilidade e adequação do instrumento de coleta e do método ao objetivo do estudo. O pré-teste realizou-se sob as mesmas circunstâncias da pesquisa e com 12 participantes que não fizeram parte da amostra final e, por fim, mostrou a confiabilidade e operacionalidade do instrumento.

Utilizou-se um instrumento que continha uma questão para aplicação de teste de associação livre de palavras (TALP) e foi solicitada a permissão para serem gravadas em áudio. Por fim, foi utilizado o roteiro para caracterização do perfil sociodemográfico e clínico do participante.

A TALP é amplamente utilizada em estudos que têm como suporte teórico a TRS, pois pos- 
sibilita a evidência de elementos semânticos de determinado grupo, mediante evocações a partir de estímulos indutores, o que possibilita conhecer a estrutura representacional. Trata-se de uma técnica projetiva que traz à consciência aspectos inconscientes por meio de manifestações de condutas de evocações ${ }^{16}$. A TALP foi aplicada no início da coleta dos dados com o intuito de evitar que a aplicação do roteiro para caracterização dos participantes eliminasse a espontaneidade das respostas. Utilizou-se como frase indutora: Diga quatro palavras ou imagens que vêm à sua cabeça quando você ouve falar em pé diabético. Após, foi solicitado ao participante que indicasse qual dos termos mencionados era o mais importante para ele. Para que a compreensão da técnica pelo participante fosse garantida, houve treinamento prévio, em que foi utilizado um estímulo indutor não relacionado ao objeto de estudo. Após verificado que houve entendimento acerca da técnica, a coleta de dados foi iniciada.

As percepções da pesquisadora foram registradas em um diário de campo imediatamente após a aplicação da TALP e os registros eram descritivos e abordavam os esclarecimentos sobre termos emitidos pelos participantes.

\section{Análise e tratamento dos dados}

Os dados empíricos foram ordenados primeiramente em tabela do Excel e, posteriormente, processados no software openEVOC 0.85 . Este programa fornece suporte ao processo de pesquisa em RS de modo alinhado com a teoria do núcleo central das RS, o que permite a identificação do sistema central a periférico das RS. O software considera a frequência simples e a ordem média de aparecimento das palavras evocadas espontaneamente que, ao serem combinados, dão origem ao quadro de quatro quadrantes que contempla e posiciona as evocações produzidas de acordo com a relevância atribuída pelos participantes da investigação.

\section{Aspectos éticos}

Em cumprimento à Resolução 466/2012 do Conselho Nacional de Saúde, o projeto de pesquisa foi aprovado no ano de 2018 pelo Comitê de Ética em Pesquisa do Hospital Universitário da Universidade Federal do Maranhão, respeitando-se os preceitos éticos de pesquisa envolvendo seres humanos.

Todos os participantes receberam informações orais e por escrito sobre o estudo e as pes- quisadoras responsáveis. O Termo de Consentimento Livre e Esclarecido foi assinado por todos os participantes e o anonimato foi garantido.

\section{Resultados}

Conhecer o perfil dos participantes do estudo auxilia numa compreensão mais ampla dos elementos representacionais dos seus modos de pensar. Dos 100 participantes da pesquisa, 51\% eram do sexo masculino e $49 \%$ do sexo feminino, com idade que varia de 33 a 92 anos (37\% tinham idade entre 60 e 69 anos). No grupo, a média de quantidade de anos de estudo era de 7,9 anos e $62,5 \%$ viviam com o cônjuge. O tempo médio de diagnóstico do DM foi de 14 anos: o tipo 1 correspondeu a 6,25\%, assim como o diabetes gestacional; e o tipo 2 foi o mais prevalente, com $87,5 \%$. Destaca-se que o grupo de pessoas abordadas com diabetes gestacional corresponde às mulheres que desenvolveram a comorbidade no período da gravidez com persistência da alteração da glicemia após o parto, mas que não se encontravam gestantes no momento da pesquisa.

A partir da análise estrutural da associação livre de palavras com a abstração da estrutura ou organização das RS do pé diabético, buscou-se elucidar o sistema central e periférico. Os resultados demonstraram que os participantes elaboraram 381 evocações com 110 expressões ou palavras distintas entre si. Após a emissão das evocações, os participantes sinalizavam a palavra que consideravam mais importante, o que possibilitou conhecer não só os conteúdos da representação, mas também a sua organização ou estrutura.

As palavras evocadas uma única vez foram desprezadas no momento da construção dos quadrantes, pois seu percentual foi considerado não significativo $(13,65 \%)$. Dessa maneira, empregou-se $86,35 \%$ do total das palavras emitidas, tornando a análise mais robusta e representativa. $\mathrm{Na}$ sequência, realizou-se o cálculo da frequência média de surgimento das evocações, dividindose o total das palavras (329 unidades de análise temáticas) pelo número das palavras distintas entre si (110), obtendo-se a frequência média de três. Para a obtenção da ordem média de evocação, o software gerou automaticamente o valor de 2,47 .

A partir do software, surge o quadro de quatro casas com as evocações ao termo indutor pé diabético, podendo-se observar a estrutura organizacional das RS, assim como a hierarquia dos 
elementos que formam seus conteúdos mentais (Quadro 1).

As 10 expressões mais mencionadas corresponderam a $60,5 \%$ do total e foram: cuidado (42), amputação (33), cura (29), medo (24), preocupação (16), incurável (12), muito ruim (12), diabetes (11), demora para cicatrizar (11) e dieta (9). A palavra "cuidado" emergiu dos discursos com o sentido de autocuidado e o seu papel fundamental na prevenção do pé diabético.

De acordo com a abordagem estrutural da TRS, as expressões que aparecem no quadrante superior esquerdo caracterizam o provável núcleo central da representação, uma vez que foram prontamente evocadas (menor ordem média de evocação) e apresentam frequência alta. $\mathrm{O}$ núcleo central é a parte mais estável e resistente às mudanças da RS e articula questões históricas, sociológicas e ideológicas, marcadas pela memória coletiva do grupo de pertença e sistema de normas a que se refere ${ }^{17}$. Os elementos que constituíram o referido quadrante foram "cura" (com o sentido de desejo/esperança em se recuperar brevemente) e "muito ruim" (como sinônimo de algo penoso), indicando que a hipótese de centralidade da RS do pé diabético encontra-se ancorada em conteúdos subjetivos e valorativos. Nas evocações, a esperança de cura esteve fortemente presente, sendo, por vezes, a única fonte de forças para a pessoa dar continuidade ao tratamento.

O grupo dos participantes do sexo masculino, com DM do tipo 2 e da faixa etária entre 60 e 69 anos possui relação especial com as duas evocações "cura" e "muito ruim", o que traz uma compreensão de que tais características os levem a perceber mais prontamente o impacto negativo que o pé diabético tem no cotidiano, conduzindo-os a gerar uma narrativa nessa linha de argumentação.

Quadro 1. Evocações das representações sociais ao termo indutor "pé diabético" obtidas a partir do software openEVOC 0.85. Maranhão, 2019.

\begin{tabular}{|c|c|c|c|c|c|}
\hline \multirow{2}{*}{\multicolumn{3}{|c|}{\begin{tabular}{|c|c|c|}
++ Frequência $\geq 3$ / Ordem de evocação $<2,47$ \\
$1^{\circ}$ Quadrante: Elementos centrais
\end{tabular}}} & \multicolumn{3}{|c|}{ + - Frequência $\geq 3$ / Ordem de evocação $\geq 2,47$} \\
\hline & & & \multicolumn{3}{|c|}{$2^{\circ}$ Quadrante: $1^{a}$ periferia } \\
\hline $9,01 \%$ & Cura & 1,83 & $12,42 \%$ & Cuidado & 2,1 \\
\hline \multirow[t]{5}{*}{$3,73 \%$} & Muito ruim & 1,75 & $10,25 \%$ & Amputação & 2,33 \\
\hline & & & $6,83 \%$ & Medo & 2,32 \\
\hline & & & $4,97 \%$ & Preocupação & 2,13 \\
\hline & & & $3,73 \%$ & Incurável & 2,17 \\
\hline & & & $3,42 \%$ & Diabetes & 2,09 \\
\hline \multicolumn{3}{|c|}{ - + Frequência $<3$ / Ordem de evocação $<2,47$} & \multicolumn{3}{|c|}{ - - Frequência $<3$ / Ordem de evocação $\geq 2,47$} \\
\hline \multicolumn{3}{|c|}{ 3० Quadrante: Zona de contraste } & \multicolumn{3}{|c|}{$4^{\circ}$ Quadrante: $2^{a}$ periferia } \\
\hline \multirow[t]{18}{*}{$0,62 \%$} & Prevenção & 1,5 & $2,8 \%$ & Demora para cicatrizar & 2,33 \\
\hline & & & $2,8 \%$ & Ferida & 2,33 \\
\hline & & & $2,8 \%$ & Curativo & 3,11 \\
\hline & & & $2,8 \%$ & Dieta & 3,44 \\
\hline & & & $2,48 \%$ & Tristeza & 3 \\
\hline & & & $1,86 \%$ & Dificuldades & 2,17 \\
\hline & & & $1,86 \%$ & Dor & 3 \\
\hline & & & $1,86 \%$ & Limitação & 3,17 \\
\hline & & & $1,86 \%$ & Remédio & 3,17 \\
\hline & & & $1,55 \%$ & Pior doença & 2,2 \\
\hline & & & $1,55 \%$ & Complicação & 2,6 \\
\hline & & & $1,24 \%$ & Sofrimento & 2 \\
\hline & & & $0,93 \%$ & Desesperado & 2 \\
\hline & & & $0,93 \%$ & Dependência & 2,33 \\
\hline & & & $0,93 \%$ & Hidratação & 2,67 \\
\hline & & & $0,93 \%$ & Nervoso & 2,67 \\
\hline & & & $0,93 \%$ & Locomoção difícil & 3 \\
\hline & & & $0,93 \%$ & Falta de cuidado & 3,33 \\
\hline
\end{tabular}

Fonte: Elaborado pelos autores (2019). 
Por outro lado, o sistema periférico consiste no complemento primordial do sistema central, no qual há regulação e adaptação dos elementos centrais às situações reais vivenciadas pelos indivíduos. Na primeira periferia, encontram-se os termos que corroboram com os elementos centrais, cujas as palavras aparecem com as maiores frequências, embora evocadas mais tardiamen$t^{17}$. Nesta investigação, o quadrante superior direito reuniu as seguintes palavras: "cuidado", "amputação", "medo", "preocupação", "incurável" e "diabetes".

O termo "cuidado" apresenta uma dimensão atitudinal relacionada à prática de cuidados consigo mesmo, o que evidencia a ideia de uma responsabilização pela sua saúde, ou seja, um cuidado como uma construção cotidiana e dependente do próprio indivíduo. Ainda na primeira periferia, nota-se a presença de palavras que dizem respeito à dimensão subjetiva da RS, envolta por preocupação com a condição e medo. Os participantes verbalizaram medo de se ferir, medo de infecção, medo de usar cadeira de rodas e medo de perder o pé.

$\mathrm{Na}$ segunda periferia (localizada no quadrante inferior direito), observam-se os termos com frequência menor e evocados mais extemporaneamente, que têm relevância no campo representacional por trazer elementos relacionados com as práticas cotidianas, o que traduz os conhecimentos dos indivíduos e guia seus comportamentos ${ }^{17}$. Tal quadrante foi formado pelas expressões/palavras: demora para cicatrizar, ferida, curativo, dieta, tristeza, dificuldades, dor, limitação, remédio, pior doença, complicação, sofrimento, desesperado, dependência, hidratação, nervoso, locomoção difícil e falta de cuidado. Nessa periferia, emergiram ideias e sentidos relacionados aos impactos negativos da condição pé diabético, assim como ao cuidado mais instrumental ou à falta de cuidado.

Percebe-se, então, nesta segunda periferia, palavras que traduzem como os participantes praticam os cuidados com os pés, compondo uma dimensão atitudinal. Aparecem ainda elementos que demonstram uma dimensão subjetiva (sofrimento, tristeza, dificuldades, dor, limitação, desesperado, pior doença) e uma dimensão relacional (dependência).

No quadrante inferior esquerdo (zona de contraste), apresentam-se os elementos com baixa frequência e rapidamente evocados, que trazem aspectos que reforçam os termos presentes no núcleo central ${ }^{17}$. Compõe esse quadrante apenas o elemento "prevenção".

\section{Discussão}

O agrupamento de palavras e as expressões evocadas para o estímulo indutor "pé diabético" e sua disposição nos quadrantes apontam que a RS do grupo estudado encontra-se fortemente ancorada em sentimento de esperança pela melhora do quadro, sentimentos negativos e reconhecimento da importância do autocuidado. O entendimento de que o pé diabético está relacionado a uma condição crônica e que se caracteriza, em menor ou maior grau, por ser um fator de limitação física com impactos no âmbito psicológico e social tem ressonância nos elementos que aparecem no sistema central e periférico dos quadrantes.

Ao considerar que o núcleo central comporta-se como a parte mais estável da representação, evidencia o pensamento coletivo e fornece elementos que compõem a identidade do agrupamento social ${ }^{13,17}$, na análise dos núcleos centrais revelados, nomeadamente "cura" e "muito ruim", pode-se inferir que o sentido atribuído fornece uma impressão inicial de certa oposição entre essas evocações, o que evidencia uma percepção antagônica da pessoa que experimenta o pé diabético. Contudo, esses dados geram pistas sobre as dificuldades diárias vivenciadas pelas pessoas para o controle da comorbidade e, concomitantemente, a conscientização adquirida pelos participantes após o acometimento do pé diabético sobre a importância do autocuidado, tanto direcionado para os cuidados com os pés quanto para outros aspectos das suas vidas, ressaltando um aspecto positivo vinculado a essa comorbidade.

O elemento central de maior evidência é "cura", e que apresenta vínculo à ideia de esperança pela mitigação da condição pé diabético. As pessoas que convivem com essa complicação, apesar de muitos já estarem habituados ao autogerenciamento do diabetes, necessitam de uma narrativa de esperança e de um pensamento positivo para manterem-se engajados com o autocuidado. Estudiosos sugerem que, no atendimento a pessoas com condições que limitam a vida, os profissionais de saúde devem auxiliá -las a encontrar o caminho da esperança que se concentra na cura, proporcionando um alicerce emocional mais realista, com alívio físico, emocional e espiritual ${ }^{18}$.

Viver com o pé diabético é um desafio permeado por nuances negativas, como demonstram os participantes deste estudo por meio do segundo elemento nuclear "muito ruim". Para as pessoas com DM, o cotidiano inclui esforços para harmo- 
nizar vários compromissos ao gerenciamento do diabetes e suas complicações. Neste contexto, há uma pressão adicional no bem-estar dessas pessoas evidenciada pelo comprometimento da qualidade de vida, tais quais aposentadoria precoce, aumento do desemprego e estresse relacionados ou ao trabalho ou aos relacionamentos sociais ${ }^{19,20}$.

Ainda conforme os princípios da abordagem estrutural da TRS ${ }^{13}$, a zona de contraste, localizada no quadrante inferior esquerdo, é formada por elementos que tiveram baixa frequência $e$ baixa ordem de evocação, entretanto, se revestem de importância para os participantes. Na pesquisa em tela, o elemento "prevenção" encontra-se na zona de contraste, ou seja, reforça e conversa com o núcleo central e, concomitantemente, retrata uma certa tensão em relação ao termo indutor "pé diabético". A "prevenção" conecta-se aos elementos "cura" e "muito ruim", o que demonstra uma certa conscientização de que aquela ação pode garantir um viver mais tranquilo $e$ evitar o aparecimento do pé diabético, excluindo, consequentemente, a necessidade da cura. Nota-se, assim, que a prevenção denota uma visão mais crítica direcionada à condição pé diabético, podendo transformar os elementos nucleares identificados.

A evocação "prevenção" indica o significado do pé diabético na concepção do grupo estudado, que possibilita ter uma conexão com a memória coletiva construída ao longo do tratamento do diabetes, no qual a equipe de saúde destaca a importância do autocuidado para evitar complicações. Entretanto, faz-nos pensar que, talvez, algumas abordagens para prevenção não têm sido suficientes para evitar complicações. Um estudo realizado na Índia sugere que, para prevenção efetiva de doença crônica, a exemplo do pé diabético, as abordagens implementadas devem incluir o público-alvo em todo processo de desenvolvimento das práticas educativas e, idealmente, essas estratégias devem ser culturalmente adaptadas para que gerem soluções inovadoras ${ }^{21}$.

Assim, uma assistência mais efetiva pode ser direcionada pela mudança de práticas nos diferentes níveis de atenção da rede de saúde, especialmente na APS, a partir da identificação dos aspectos relacionados às ações de prevenção do pé diabético, como a avaliação dos pés dos pacientes de risco, identificando-se indivíduos mais vulneráveis, o que previnirá e reduzirá complicações posteriores?.

Os elementos que aparecem na primeira periferia, no quadrante superior direito (Quadro 1), demonstram como o grupo que apresenta pé diabético se adapta às situações do cotidiano. As palavras "cuidado", "incurável" e "diabetes" expressam um ser ciente que apresenta uma condição crônica e a necessidade constante de autocuidado, mas vivem diuturnamente com "medo" e "preocupação" por saber que se trata de uma comorbidade que pode evoluir para complicações indesejáveis e devastadoras, como a "amputação"dos membros.

A maior complicação do pé diabético incorre em amputações, com custos elevados para o Sistema Único de Saúde. Essa foi uma preocupação revelada por parte dos entrevistados. Em estudo realizado por pesquisadores da Universidade de Goiás, Universidade do Estado do Rio de Janeiro e Universidade Federal do Rio Grande do Sul, entre 2014 e 2017 demonstrou que, dos custos médicos anuais totais estimados para todo o Brasil, tomando como base o ano de $2014,85 \%$ dos custos estavam relacionados ao tratamento de pacientes com pé neuroisquêmico com úlcera ${ }^{22}$.

O termo de maior relevância no quadrante superior direito foi "cuidado", e emergiu dos discursos com o sentido de autocuidado, demonstrando a conscientização dos participantes sobre o papel fundamental do autogerenciamento da díade diabetes/pé diabético. O DM é uma doença complexa que requer uma autogestão eficaz. Estudiosos afirmam que, quanto maior a autonomia dos pacientes, melhor é o autogerenciamento e maior é a autoeficácia da pessoa com diabetes. Assim, é de extrema importância que a equipe de saúde desenvolva comunicação proativa com as pessoas que apresentam pé diabético ou têm risco para desenvolvê-lo com o objetivo de estimular o senso de autonomia e promover um melhor autogerenciamento da condição pé diabético, incrementando essa abordagem com acordos comportamentais que respeitem as preferências das pessoas e as apoiem na adoção de práticas de autocuidado ${ }^{23,24}$.

As evocações "limitação", "dependência" e "locomoção difícil" se conectam e denotam a dimensão imagética da $\mathrm{RS}^{12} \mathrm{em}$ relação ao objeto explorado. Essas evocações indicam aspectos negativos, com repercussões no cotidiano do grupo explorado. Habitualmente, o aparecimento do pé diabético traz limitações, com necessidade de ajustes para a vida fluir, como, por exemplo, o uso de auxílio-locomoção e exigência de suporte de terceiros para cuidados antes feitos por si só. $\mathrm{O}$ surgimento dessas evocações, na segunda periferia, aparenta estar ancorada na ideia socialmente disseminada de que o aparecimento do pé diabético está associado ao uso inevitável de dispo- 
sitivos ortopédicos e à marcha necessariamente prejudicada ${ }^{25}$.

Experienciar uma comorbidade crônica é um processo multidimensional, dinâmico, complexo e cíclico que exige a elaboração de alguns atributos, como aceitação, enfrentamento, autogestão, dentre outros, que, dependente de como essas propriedades atuam, podem emergir modos diversos de viver que vão desde a recusa até um novo normal. Compreender como esses problemas se processam é importante para a equipe de saúde dos diferentes níveis de atenção, pois pode contribuir para um atendimento centrado na pessoa e para uma análise mais ampla e uniforme sobre como é viver com pé diabético, servindoe que serve como um guia para o desenvolvimento de intervenções apropriadas para facilitar esse processo e amenizar os impactos negativos ${ }^{26}$.

No estudo em tela, verificou-se que o tempo médio de diagnóstico de DM foi de 14 anos e os participantes relataram medo, especialmente de amputação do pé. Pesquisa realizada nos Estados Unidos da América descobriu que pacientes com pé diabético apresentaram maior medo de amputação do que morte, sendo quee uma das variáveis com maior associação com o medo foi a duração do $\mathrm{DM} \geq 10$ anos, encontrando-se em consonância com este estudo. A pesquisa apontou que pacientes com feridas crônicas nas pernas experimentam sentimentos negativos associados a níveis mais elevados de depressão e quadro de ansiedade que geram estresse psicológico de difícil gerenciamento ao longo do tempo. Conhecer os medos das pessoas com pé diabético fornece pistas à equipe de saúde de como experimentam a comorbidade cotidianamente e contribuem para intervenções mais precoces com o intuito de prevenir alterações psicológicas mais graves ${ }^{27,28}$.

Os achados deste estudo são importantes para subsidiar o acompanhamento das pessoas que vivem com diabetes na APS, pois em municípios de pequeno porte podem ser escasso ou inexistente os serviços especializados de suporte no tratamento do pé diabético.

Os elementos "sofrimento", "desesperado" e "tristeza" suscitam um debate sobre a vivência com o pé diabético, percebido pelo grupo explorado como experiência angustiante fundamentada na constatação das limitações impostas pela comorbidade que impactam na vida social, laboral e psicológica. A partir das limitações, surge o sofrimento, uma vez que para lidar diariamente com essas questões faz-se necessária uma grande mobilização de energia que pode gerar desgaste psíquico.
A percepção do estado de saúde que vem junto à evocação induzida pela pesquisa, proporciona ao paciente uma maior consciência de seu estado, atuandoe atua de forma a reforçar sua educação em saúde e, consequentemente, o autocuidado. Considerando que Os cuidados diários necessários ao tratamento do diabetes são realizados pela própria pessoa ou seus familiares, logo as metodologias educativas participativas auxiliam nessa perspectiva ${ }^{1}$.

Descobertas de pesquisa apontam que o pé diabético traz prejuízos acentuados ao funcionamento psicológico e emocional, trazendoo que gera significados distintos ao âmbito social, cognitivo e individual, exigindo, e exige por parte dos profissionais de saúde, humanização, competências e habilidades específicas para conceber intervenções plurais que abordem o sofrimento psicológico enfrentado pelas pessoas que apresentam essa complicação ${ }^{29}$.

Consoante os fundamentos da perspectiva estrutural da TRS, o sistema periférico está associado à vivência concreta, engloba os elementos de transição e renova o núcleo central ${ }^{17}$. Esse processo alavanca a mudança da realidade social, o que gera contributos que subsidiam escolhas comportamentais conscientes e tomada de decisão mais acertada por parte das pessoas com pé diabético ou com risco para desenvolvê-lo.

\section{Limitações do estudo}

$\mathrm{O}$ estudo apresenta algumas limitações. $\mathrm{O}$ método de análise escolhido foi o Programa openEVOC que foca na identificação dos elementos estruturantes das RS. Entretanto, acredita-se que pesquisas adicionais que utilizem a análise do discurso possam contribuir para uma melhor compreensão do pé diabético como fenômeno psicossocial ou a realização de pesquisas com triangulação metodológica.

Acrescenta-se ainda como limitação, a realização da coleta de dados nos serviços de saúde, o que pode ter gerado algum tipo de desconforto nos participantes, não sendo possível afirmar se os elementos constituintes das RS sobre pé diabético seriam os mesmos em outros cenários, o que constitui, assim, em uma lacuna de oportunidade para estudos futuros.

Embora essas limitações tenham sido identificadas, acredita-se que o estudo em tela seja útil para profissionais de saúde que desenvolvem atividades junto a pessoas com pé diabético ou risco para desenvolvê-lo nos diferentes pontos da rede de atenção, em especial a atenção primária, 
pois traz novo conhecimento, sob a perspectiva psicossocial, do fenômeno explorado.

\section{Considerações finais}

A abordagem teórico-metodológica possibilitou o ordenamento das evocações nos quatro quadrantes, o que possibilita identificar os elementos estruturantes que orientam a formação das RS do pé diabético entre pessoas com DM. Os resultados acrescentam ao conhecimento atual os elementos constituintes da RS do pé diabético, presentes no núcleo central, e referem-se às evocações "cura” e "muito ruim", e revelam que viver com pé diabético é desafiador, em um nível individual, mas há uma esperança de cura que se constitui em mola propulsora na busca cotidiana do "cuidado", elemento este que compõe a primeira periferia, e que corrobora com o núcleo central. O cotidiano do cuidado, por sua vez, está intrínseco ao nível primário de atenção à saúde, ancorado nos princípios da APS, a qual deve estar preparada formativa e operacionalmente para a prevenção e cuidado dos pacientes com diabetes de maneira integral.

Neste sentido, essa pode ser uma área próspera para o desenvolvimento de programas educacionais que estimulem a autorresponsabilidade pelos seus cuidados, supram demandas psicossociais e aumente a percepção de suporte social das pessoas com pé diabético ou com risco para desenvolvê-lo. Paralelamente, faz-se necessária a criação de programas de formação de profissionais de saúde com o intuito de melhor prepará -los para lidar com questões psicossociais trazidas por este público-alvo e para planejar e implementar intervenções sensíveis a uma compreensão sociocultural e ao modo como as pessoas interagem socialmente, o que qualifica ainda mais os serviços de saúde, especialmente os ligados a APS, e contribui para o fortalecimento de um cuidado coordenado e integral às pessoas com diabetes.

O dinamismo das RS possibilita a compreensão de como essas estruturas mentais guiam as crenças, opiniões e comportamentos dessas pessoas, contribuindo, assim, para a concepção de intervenções mais específicas e inovadoras direcionadas para o estímulo da autonomia, o suporte emocional e psicológico e que apoie a adoção de comportamentos saudáveis que conduzam a resultados positivos na saúde dos pés.

É de fundamental importância que os profissionais de saúde criem oportunidades dentro dos espaços de atendimento dos serviços primários e especializados para que seja praticável uma escuta qualificada, que explore as crenças e percepções das pessoas sobre a sua experiência com o pé diabético, de modo que suas intervenções sejam capazes de sensibilizá-los, desafiá-los a refletir sobre possíveis crenças equivocadas e os levem a ressignificar sua condição, com repercussão favorável no seu autocuidado e influenciando-os a fazer escolhas comportamentais eficientes.

\section{Colaboradores}

GSG Lopes participou na concepção, delineamento, análise e interpretação dos dados, redação e revisão crítica do artigo. ILTP Rolim participou na concepção, delineamento, análise e interpretação dos dados, redação e, na condição de orientadora da pesquisa, participou como revisora de todas as etapas na elaboração do artigo. RS Alves, TRRF Pessoa, ER Maia, MSV Lopes, APP Morais e RCS Queiroz participaram da redação e revisão crítica do artigo. 


\section{Agradecimentos}

À Coordenação de Aperfeiçoamento de Pessoal de Nível Superior-Brasil (CAPES), pelo financiamento da investigação através do código 001.

\section{Referências}

1. Brasil. Ministério da Saúde (MS). Secretaria de Atenção à Saúde. Manual do pé diabético: estratégias para o cuidado da pessoa com doença crônica. Brasília: MS; 2016.

2. Schaper NC, Netten JJV, Apelqvist J, Bus SA, Hinchliffe RJ, Lipsky BA. IWGDF Guidelin es on the prevention and management of diabetic foot disease. Netherlands: The International Working Group on the Diabetic Foot [Internet]. 2019 [cited 2020 Nov 14]. Available from: https://iwgdfguidelines.org/wp-content/uploads/2019/05/IWGDF-Guidelines-2019.pdf

3. Alexiadou K, Doupis J. Management of diabetic foot ulcers. diabetes ther [serial on the Internet]. 2012 [cited 2020 Jul 26]; 3(1):1-15. Available from: https:// www.ncbi.nlm.nih.gov/pmc/articles/PMC3508111/

4. Jeffcoate WJ, Vileikyte L, Boyko EJ, Armstrong DG, Boulton AJM. Current challenges and opportunities in the prevention and management of diabetic foot ulcers. Diabetes Care 2018; 41(4): 645-652.

5. Zhang P, Lu J, Jing Y, Tang S, Zhu D, Bi Y. Global epidemiology of diabetic foot ulceration: a systematic review and meta-analysis. Ann Med [serial on the Internet]. 2017 [cited 2020 Nov 14]; 49(2): 106-116. Available from: https://www.tandfonline.com/doi/ful 1/10.1080/07853890.2016.1231932

6. Namgoong S, Jung S, Han SK, Jeong SH, Dhong ES, Kim WK. Risk factors for major amputation in hospitalised diabetic foot patients. Int Wound $J$ [serial on the Internet]. 2016 [cited 2020 Nov 14]; 13(Supl. 1): 13-19. Available from: https://onlinelibrary.wiley. com/doi/full/10.1111/iwj.12526

7. Seguel G. ¿Por qué debemos preocuparnos del pie diabético? Importancia del pie diabético. Rev Med Chile [periódico de Internet]. 2013 [Consultado $2020 \mathrm{Nov}$ 14]; 141(11):1464-1469. Disponible en: https://scielo. conicyt.cl/pdf/rmc/v141n11/art14.pdf

8. Sothornwit J, Srisawasdi G, Suwannakin A, Sriwijitkamol A. Decreased health-related quality of life in patients with diabetic foot problems. Diabetes Metab Syndr Obes [serial on the Internet]. 2018 [cited 2020 Nov 14]; 7(11): 35-43. Available from: https://www. ncbi.nlm.nih.gov/pmc/articles/PMC5846758/

9. Fernandes FCGM, Santos EGO, Morais JFG, Medeiros LMF, Barbosa IR. The care of feet and the prevention of ulcers in diabetic patients in Brazil. Cad Saude Colet [serial on the Internet]. 2020 [cited 2020 Nov 14]; 28(2): 302-310. Available from: https://www. scielo.br/pdf/cadsc/v28n2/1414-462X-cadsc-1414462X202028020258.pdf

10. Silva PS, Vieira CSA, Gomes LMX, Barbosa TLA. Degree of risk of diabetic foot in primary health care. Rev Enferm UFSM [serial on the Internet]. 2020 [cited 2020 Nov 14]; 10(e78): 1-16. Available from: https:// periodicos.ufsm.br/reufsm/article/view/42614/html

11. Ramirez-Perdomo C, Perdomo-Romero A, Rodríguez-Vélez M. Knowledge and practices for the prevention of the diabetic foot. Rev Gaucha Enferm [serial on the Internet]. 2019 [cited 2020 Nov 14]; 40(e20180161): 1-8. Available from: https://www.scielo.br/pdf/rgenf/v40/1983-1447-rgenf-40-e20180161. pdf 
12. Moscovici S. Representações sociais: investigações em psicologia social. Petrópolis: Vozes; 2015.

13. Abric J. Pratiques sociales et représentations. Paris: Presses Universitaires de France; 1994.

14. Carvalho MCMP, Queiroz ABA, Ferreira MA, Moura MAV, Pinto CB, Vieira BDG. Ineffectiveness of information and access to health services: vulnerability to human papillomavirus. Int Nurs Rev [serial on the Internet]. 2019 [cited 2020 Nov 14]; 66(2): 280-289. Available from: https://onlinelibrary.wiley.com/doi/ abs/10.1111/inr.12500

15. Moura LM, Shimizu HE. Social representations of health and illness of municipal health counselors. Physis [serial on the Internet]. 2017 [cited 2020 Nov 14]; 27(1): 103-125. Disponível em: https://www.scielo.br/ pdf/physis/v27n1/0103-7331-physis-27-01-00103.pdf

16. Nóbrega SM, Coutinho MDPL. O teste de associação livre de palavras. In: Coutinho MPL, Lima AS, Oliveira FB, Fortunato ML. Representações sociais: abordagem interdisciplinar. João Pessoa: Universitária; 2003. p. 67-77.

17. Sá CP. Estudos de psicologia social: história, comportamento, representações e memória. Rio de Janeiro: EdUERJ; 2015.

18. Stuart B, Danaher T, Awdish R, Berry L. Finding hope and healing when cure is not possible. Mayo Clin Proc 2019; 94(4): 677-685.

19. Hansen UM, Olesen K, Browne JL, Skinner TC Willaing I. A call for inclusion of work-related diabetes distress in the spectrum of diabetes management: results from a cross-sectional survey among working people with type 1 diabetes. Diabetes Res Clin Pract 2018 Jun; 140: 139-147.

20. Nexø MA, Pedersen J, Cleal B, Bjorner JB. Increased risk of long-term sickness absence, lower rate of return to work and higher risk of disability pension among people with type 1 and type 2 diabetes mellitus: a Danish retrospective cohort study with up to 17 years follow-up. Diabet Med 2019 Dec 6; 37(11):1861-1865.

21. Andrade NS, Espey DK, Hall ME, Bauer UE. A holistic approach to chronic disease prevention: good health and wellness in indian country. Prev Chronic Dis [serial on the Internet]. 2019 [cited 2020 Nov 14]; 16(E98):1-5. Available from: https://www.cdc.gov/pcd /issues/2019/pdf/19_0081.pdf

22. Bahia L. O alto custo do pé diabético no Brasil. Sociedade Brasileira de Diabetes [Internet]. 2018 [acessado 2020 nov 12]. Disponível em: https://www.diabetes. org.br/publico/ultimas/1609-o-alto-custo-do-pe-diabetico-no-brasil

23. Bullen B, Young M, McArdle C, Ellis M. Overcoming barriers to self-management: the person-centred diabetes foot behavioural agreement. Foot (Edinb) 2019 Mar; 38: 65-69.
24. Robinson SA, Zocchi MS, Netherton D, Ash A, Purington CM, Connolly SL, Vimalananda VG, Hogan TP, Shimada SL. Secure messaging, diabetes self-management, and the importance of patient autonomy: a mixed methods study. J Gen Intern Med [serial on the Internet]. 2020 [cited 2020 Nov 14]. Available from: https://escholarship.umassmed.edu/cgi/viewcontent. cgi? article $=2358 \&$ context $=$ qhs_pp

25. Coffey L, Mahon C, Gallagher P. Perceptions and experiences of diabetic foot ulceration and foot care in people with diabetes: A qualitative meta-synthesis. Int Wound J 2019 [cited 2020 Nov 12]; 16(1):183-210. Avaliable from: https://onlinelibrary.wiley.com/ doi/ full/10.1111/iwj.13010

26. Ambrosio L, García JMS, Fernández MR, Bravo SA, Ayesa SDDC, Sesma MEU, Caparrós N, Portillo MC. Living with chronic illness in adults: a concept analysis. J Clin Nurs 2015; 24(17-18): 2357-2367.

27. Wukich DK, Raspovic KM, Suder NC. Patients with diabetic foot disease fear major lower-extremity amputation more than death. Foot Ankle Spec 2018; 11(1):17-21

28. Platsidaki E, Kouris A, Christodoulou C. Psychosocial aspects in patients with chronic leg ulcers. Wounds [serial on the Internet]. 2017 Oct [cited 2020 Nov 14]; 29(10): 306-310. Available from: https://www.woundsresearch.com/article/psychosocial-aspects-patients-chronic-leg-ulcers

29. Vileikyte L, Crews RT, Reeves ND. Psychological and biomechanical aspects of patient adaptation to diabetic neuropathy and foot ulceration. Curr Diab Rep 2017; 17(11):109.

Artigo apresentado em 17/07/2020

Aprovado em 26/02/2021

Versão final apresentada em 28/02/2021

Editores-chefes: Romeu Gomes, Antônio Augusto Moura da Silva 
